\title{
Persistent Bloodstream Infection with $P$. aeruginosa in a Patient with an Aortic Root Graft and a New Diagnosis of Lung Cancer
}

Chen X, Ramirez A and Al Achkar M*

Department of Family Medicine, Indiana University, USA

*Corresponding author: Al Achkar M, Department of Family Medicine, Indiana University, 1520 N. Senate Ave, Indianapolis, IN 46205, USA, Tel: 3179628893; E-mail: alachkar@iupui.edu

Received date: July12, 2016; Accepted date: July 20, 2016; Published date: July 26, 2016

Copyright: (C) 2016 Chen X, et al. This is an open-access article distributed under the terms of the Creative Commons Attribution License, which permits unrestricted use, distribution, and reproduction in any medium, provided the original author and source are credited.

\begin{abstract}
Pseudomonas aeruginosa is the third most common gram-negative pathogen causing bloodstream infection (BSI) and is associated with an elevated mortality rate of up to $42 \%$. The primary sources of infection are the pulmonary system, indwelling catheters, urinary tract, and soft tissue. BSI caused by vascular graft infection is $1-6 \%$ depending on the location of the graft. There is increasing prevalence of resistance of $P$. aeruginosa to antimicrobial agents, and oral agents are usually not considered as treatment of choice for $P$. aeruginosa BSI. Here, we report a case of persistent BSI with $P$. aeruginosa with the aortic graft as the primary source of infection. Patient did not go through aortic graft extraction due to new diagnosis of end stage lung cancer but was successfully managed by oral suppression therapy after failing a prolonged course of IV antibiotic therapy.
\end{abstract}

Keywords: P. aeruginosa; Aortic graft; Blood stream infection

\section{Introduction}

$P$. aeruginosa is the third most common gram-negative pathogen causing Bloodstream Infections (BSI), and it is a worldwide problem [1-8]. A nationwide surveillance study, Surveillance and Control of Pathogens of Epidemiological Importance (SCOPE), performed in 49 hospitals between 1995 and 2002, reported that pseudomonas accounted for $4 \%$ of total hospital acquired BSI [5]. In intensive care units, the proportion of gram-negative BSI due to $P$. aeruginosa is even higher. The incidence of $P$. aeruginosa BSI increased exponentially with age and was higher in male patients than in female patients, especially after the age of 50 [9]. It was reported in a population-based study in the United States that up to $78 \%$ of cases of $P$. aeruginosa related BSIs were either hospital acquired or health care associated, while $12 \%$ were community acquired [4]. The 30-day mortality of $P$. aeruginosa bacteremia was as high as $39 \%$ in one investigation [10].

Risk factors for $P$. aeruginosa bacteremia include neutropenia or other immunodeficiency, advanced age, pancreatobiliary tract disease, severe burns, an indwelling central venous or urinary catheter, recent antibiotic use, and traumatic wounds. It was demonstrated in a retrospective cohort study that lung cancer and antibiotic use were two of the major predictors for pseudomonas BSI in non-neutropenic patients with a solid tumor [11]. Common primary sites of infection causing $P$. aeruginosa BSI include: respiratory, central venous catheters, urinary tract, and soft tissue $[4,12,13]$.

\section{Case}

Here we report a case of persistent BSI with $P$. aeruginosa in the presence of an aortic graft and a new diagnosis of lung cancer. Patient is a 65-year-old male who presented to the emergency department with intermittent fevers, progressive dyspnea, productive cough, and generalized fatigue. His past medical history included remote history of aortic transection after a motor vehicle accident status post aortic grafting (placed in 2002), Congestive Heart Failure (CHF) with Implantable Cardioverter Device (ICD) secondary to dilated cardiomyopathy, Hepatitis C, Coronary artery disease, hyperlipidemia, Chronic Obstructive Pulmonary Disease (COPD), and GastroEsophageal Reflux Disease (GERD). He had a 2-3 pack-per-day smoking history for 50 years yet recently quit smoking about 6 months prior to presentation. The patient was admitted to the hospital multiple times in the past year for prolonged periods of dyspnea, with the most recent hospitalization being for a COPD exacerbation about 2 months ago.

Vitals signs at the time of admission revealed a temperature of 100.8F; HR:101; RR:27; BP:119/39; and 95\% on $2 \mathrm{~L} \mathrm{O}_{2}$ (baseline). Initial exam showed that the patient was alert and oriented. He had slight tachycardia and had no murmur on cardiac auscultation. He had slight wheezing of the lungs and had no lower extremity edema. Initial labs revealed a leukocytosis with a white blood cell (WBC) count of 15 $\mathrm{k} / \mathrm{mm}^{3}$. Initial basic metabolic panel showed a creatinine of $1.5 \mathrm{mg} / \mathrm{dl}$, which was slightly elevated above his baseline. Having met criteria for systematic inflammatory response syndrome (SIRS) with unknown source of infection, the patient was started on broad spectrum antibiotics with Vancomycin and Piperacillin with Tazobactam. Urine culture and blood culture were drawn prior to initiation of antibiotic therapy.

The urine culture remained negative and the blood culture resulted positive for $P$. aeruginosa. Repeat blood culture on day 2 (day 0 is defined as the day of admission and the first dose of antibiotics) stayed positive for $P$. aeruginosa in $1 / 4$ bottles and were sensitive to Piperacillin with Tazobactam but resistant to Cefepime and Meropenem by E-testing. On day 5, the patient had been afebrile for 72 hours, and his leukocytosis had resolved. He was discharged with continuous outpatient IV Piperacillin with Tazobactam via a peripherally inserted central catheter (PICC) line. Blood cultures which were drawn at discharge remained positive for $P$. aeruginosa in $2 / 4$ bottles. Piperacillin with Tazobactam was continued, and repeated blood cultures on day 9 still remained positive for $P$. aeruginosa. At this 
time, the patient was contacted and readmitted to the hospital for further investigation of his persistent BSI with $P$. aeruginosa.

During the readmission, his Piperacillin with Tazobactam was switched to Cefepime based on a recent sensitivity study, and he also received one dose of Tobramycin. Given concerns for PICC line and ICD lead infection, both his PICC line and ICD were removed and were both culture negative. Nevertheless, subsequent blood cultures remained positive for $P$. aeruginosa. Trans Esophageal Echocardiogram (TEE) failed to reveal any signs of endocardial vegetation. CT of the chest did not show any signs of infection, but did however, show a new right hilar and mediastinal adenopathy concerning for lung cancer. Lung biopsy confirmed the diagnosis of small cell carcinoma. Further work up for persistent BSI included a WBC tag study which also failed to reveal any focal activity. Finally, a positron emission tomography (PET) scan showed increased activity in the mid-thoracic aortic stent concerning for aortic graft infection. Considering his status of cancer, the risk of surgical intervention for possible aortic graft infection was considered significantly higher than the benefit and deemed inappropriate. By this time, patient's blood culture had remained positive despite 6 weeks of IV antibiotics. Finally patient declined further IV antibiotics and he was subsequently discharged on oral ciprofloxacin for suppression therapy. His follow-up blood cultures finally turned negative and have remained negative until the last follow-up performed on 01/06/2015.

\section{Discussion}

In this case, our patient had multiple risk factors for BSI with $P$. aeruginosa including recent hospitalization, antibiotics use, lung cancer, and presence of an aortic graft. It has been demonstrated in a retrospective cohort study that lung cancer is one of the major predictors for pseudomonas BSI in non-neutropenic patients with solid tumor [11]. The connection between his lung cancer and the BSI with pseudomonas was not clear, but his history of smoking and state of active lung cancer definitely placed him at a higher risk for pseudomonas colonization in his respiratory tract. Furthermore, the presence of bacteremia made the patient a poor candidate to receiving chemotherapy, which would further dampen his immune system.

After exhaustive studies, the primary source of for $P$. aeruginosa causing the bacteremia was considered to be a persistent aortic graft infection. Prosthetic aortic graft insertion is complicated by infection in $0.5-6 \%$ of cases depending on the location of the grafts [14-16]. Infection likely occurs in most cases at the time of graft implantation and at the time of a subsequent procedure that involves the graft [16] Staphylococcus species are the most commonly implicated causative organisms [14-17] with Staphylococcus aureus more likely in early infection, and coagulase-negative staphylococci such as Staphylococcus epidermidis more likely in late infections $[18,19]$. Other microorganisms, including Escherichia coli, Pseudomonas, Klebsiella, Proteus, Enterobacter, Bacteroides, nonhemolytic streptococci, and occasionally yeast account for most of the remainder [18].

The diagnosis of aortic graft infection is usually made on the basis of clinical findings, supported by radiological imaging and microbiological testings [14]. Clinical manifestations of aortic graft infection may vary based on when the graft was initially placed. The presentation of late-onset infections (those occurring more than 4 months after surgery) tends to be more subtle with non-specific signs and symptoms in the absence of fever [14]. In this case, our patient had a low-grade fever at initial presentation and had some non-specific signs of symptoms including fatigue. CT is the imaging modality of choice for diagnosis [20,21]. Other investigations include technetium-99m-hexametazime-labeled leucocyte scanning, which has a high sensitivity [21,22] and sinography, which can determine whether a draining sinus extends to the graft. In our case, both the CT study and leucocyte tagged scanning test were not able to identify the aortic graft as the source of infection. Finally, the PET scan showed increased activity in the mid-thoracic aortic stent confirming the diagnosis of aortic graft infection.

Generally, the treatment of an infected graft material is to have it removed completely. Antibiotics without intervention of the infected aortic graft have rarely been advocated as a sole therapy. In special situations, a patient's significant comorbidities can preclude the excision of the graft. Long-term conservative nonresectional management of aortic graft infections with antibiotics use is the optimal management. A retrospective cohort study performed in two large hospitals in patients who were found to have endograft infection after endovascular repair of and abdominal or thoracic aortic aneurysms found that there was no significant difference in mortality between the conservatively and the surgically-managed group [23]. This study was limited by its small sample size of only 11 patients. Another study done in 2011 reported that 5 patients who had an aortic graft infection and significant other comorbidities and went through conservative management with antibiotics all survived at a mean of 32 months and a maximum period of 6 years without complications $[24,25]$. In this case, considering the patient's end stage cancer, conservative management with long-term antibiotics was used to manage the persistent BSI with pseudomonas which resulted in an optimal result. At the time of the preparation of this manuscript, the blood culture in this patient remains negative 9 months after the initial presentation, and he has had no direct complications from his BSI.

\section{References}

1. Al-Hasan MN, Wilson JW, Lahr BD, Eckel-Passow JE, Baddour LM (2008) Incidence of Pseudomonas aeruginosa bacteremia: a populationbased study. Am J Med 121: 702-708.

2. National nosocomial infections surveillance (NNIS) report, data summary from October 1986-April 1996 (1996) A report from the NNIS System. Am J Infect Control 24: 380-388.

3. Vidal F, Mensa J, Almela M, Martinez JA, Marco F, et al. (1996) Epidemiology and outcome of Pseudomonas aeruginosa bacteremia, with special emphasis on the influence of antibiotic treatment: analysis of 189 episodes. Arch Intern Med 156: 2121-2126.

4. Sifuentes-Osornio J, Gonzalez R, Ponce-de-Leon A, Guerrero MDL (1997) Epidemiology and prognosis of Pseudomonas aeruginosa bacteremia in a tertiary care center. Rev Invest Clin 50: 383-388.

5. Wisplinghoff H, Bischoff T, Tallent SM, Seifert H, Wenzel RP, et al. (2004) Nosocomial bloodstream infections in US hospitals: analysis of 24,179 cases from a prospective nationwide surveillance study. Clin Infect Dis 39: 309-317.

6. Chatzinikolaou I, Abi-Said D, Bodey GP, Rolston KV, Tarrand JJ, et al. (2000) Recent experience with Pseudomonas aeruginosa bacteremia in patients with cancer: retrospective analysis of 245 episodes. Arch Intern Med 160: 501-509.

7. McCarthy K (2015) Pseudomonas aeruginosa: evolution of antimicrobial resistance and implications for therapy. Semin Respir Crit Care Med 36: 44-55.

8. Magill SS, Edwards JR, Bamberg W, Beldavs ZG, Dumyati G, et al. (2014) Emerging infections program healthcare-associated infections and antimicrobial use prevalence survey team: multistate point-prevalence survey of healthcare-associated infections. N Engl J Med 370: 1198-1208. 
Citation: Chen X, Ramirez A, AlAchkar M (2016) Persistent Bloodstream Infection with P. aeruginosa in a Patient with an Aortic Root Graft and a New Diagnosis of Lung Cancer . Lung Dis Treat 2: 112. doi:10.4172/2472-1018.1000112

Page 3 of 3

9. Uslan DZ, Crane SJ, Steckelberg JM, Cockerill FR, Sauver JL, et al. (2007) Age- and sex-associated trends in bloodstream infection: a populationbased study in Olmsted County, Minnesota. Arch Intern Med 167: 834-839.

10. Kang CI, Kim SH, Kim HB, Park SW, Choe YJ, et al. (2003) Pseudomonas aeruginosa bacteremia: risk factors for mortality and influence of delayed receipt of effective antimicrobial therapy on clinical outcome. Clin Infect Dis 37: 745-751.

11. Joo EJ, Kang CI, Ha YE, Kim J, Kang SJ, et al. (2011) Clinical predictors of Pseudomonas aeruginosa bacteremia among gram-negative bacterial infections in non-neutropenic patients with solid tumor. J Infect 63: 207-214.

12. Chen SC, Lawrence RH, Byth K, Sorrell TC (1993) Pseudomonas aeruginosa bacteraemia: Is pancreatobiliary disease a risk factor?. Med J Aust 159: 592-597.

13. Pseudomonas aeruginosa infections associated with transrectal ultrasound-guided prostate biopsies-Georgia (2005) Centers for Disease Control and Prevention (CDC). MMWR Morb Mortal Wkly Rep 55: 776-777.

14. FitzGerald SF, Kelly C, Humphreys H (2005) Diagnosis and treatment of prosthetic aortic graft infections: confusion and inconsistency in the absence of evidence or consensus. J Antimicrob Chemother 56: 996-999.

15. Liekweg WG Jr, Greenfield LJ (1977) Vascular prosthetic infections: collected experience and results of treatment. Surgery 81: 335-342.

16. Seeger JM (2000) Management of patients with prosthetic vascular graft infection. Am Surg 66: 166-177.
17. O'Hara PJ, Hertzer NR, Beven EG, Krajewski LP (1986) Surgical management of infected abdominal aortic grafts: review of a 25-year experience. J Vasc Surg 3: 725-731.

18. Valentine RJ (2001) Diagnosis and management of aortic graft infection. Seminars Vasc Surg 14: 292-301.

19. Kaebnick HW, Bandyk DF, Bergamini TW, Towne JB (1987) The microbiology of explanted vascular prostheses. Surgery 102: 756-762.

20. Orton DF, LeVeen RF, Saigh JA, Culp WC, Fidler JL, et al. (2000) Aortic prosthetic graft infections: radiologic manifestations and implications for management. Radiographics 20: 977-993.

21. Modrall JG, Clagett GP (1999) The role of imaging techniques in evaluating possible graft infections. Semin Vasc Surg 12: 339-347.

22. Liberatore M, Iurilli AP, Ponzo F, Prosperi D (1998) Clinical usefulness of technetium-99 m-HMPAO-labeled leukocyte scan in prosthetic vascular graft infection. J Nucl Med 39: 875-879.

23. Cernohorsky P, Reijnen MM, Tielliu IF, van Sterkenburg SM, van den Dungen JJ, et al. (2011) The relevance of aortic endograft prosthetic infection. J Vasc Surg 54: 327-333.

24. Lawrence PF (2011) Conservative treatment of aortic graft infection. Semin Vasc Surg 24: 199-204.

25. Hasse B, Husmann L, Zinkernagel A, Weber R, Lachat M, et al. (2013) Vascular graft infections. Swiss Med Wkly 143: w13754. 